\title{
Insight \\ Finding and Filling the "Cracks" In Resistance Surfaces for Least-cost Modeling
}

\author{
$\underline{\text { Kristina Rothley }}^{l}$
}

\begin{abstract}
Least-cost modeling is an increasingly popular method used to measure the effective distance between habitat patches and to assess the connectivity of existing landscapes or potential reserves. For least-cost models to be reliable and credible, however, the validity of input data must be demonstrated. Least-cost modelers must also exercise extreme caution when using any GIS-based analysis of this kind. Technical issues associated with the raster-based representation of spatial data may introduce errors in otherwise correct data that nullify results. In this paper, I address the potential presence of "cracks" in the resistance input layer of least-cost modeling exercises. Cracks result when narrow, costly features, such as roads or train tracks, are represented in raster form. These cracks lead to the erroneous identification of nonexistent "shortcuts" across truly expensive barriers. In this paper, I use a hypothetical example to explain resistance-layer cracks, how they occur, and the errors they generate with respect to least-cost modeling. I then present a simple algorithm to reveal the location of cracks and one approach to filling the cracks. Finally, these methods are demonstrated on a real data set in which more than $1 \%$ of the raster cells are shown to be cracks. The negative repercussions of faulty resistance layers when conducting least-cost modeling can not be overstated. On the one hand, unimportant or nonexistent least-cost paths on a landscape may be mislabeled as important. As a result, conservation resources committed to protecting these paths will be wasted. Conversely, truly critical corridors may be overlooked, jeopardizing the organisms that rely on them.
\end{abstract}

Key Words: connectivity analysis, cracks, least-cost modeling, resistance layers.

\section{INTRODUCTION}

Connectivity is the degree to which the landscape facilitates or impedes the movement of organisms among patches (Taylor et al. 1993, Tischendorf and Fahrig 2000a). Conservation scientists generally agree that greater connectivity is a desirable quality for nature reserves (Knaapen et al. 1992, Adriaensen et al. 2003). Reserves that are more effectively connected may support larger populations that are less likely to succumb to random local extinction events than those in less-connected reserves. Greater exchange of individuals between populations can increase overall genetic heterozygosity and minimize the harmful effects of the loss of genetic variation (Allendorf and Leary 1986, Soulé and Simberloff 1986). Some species that inhabit habitat fragments must disperse to and colonize new sites for long-term regional persistence (Briers 2002).

Least-cost modeling is an increasingly popular method (Adriaensen et al. 2003) used to measure the effective distance between habitat patches and to assess the connectivity of existing landscapes or potential reserves (Meegan and Maehr 2002, Larkin et al. 2004). This functional approach (Tischendorf and Fahrig 2000b) is distinctly different from and potentially preferable to structural measures of habitat isolation, such as contagion metrics. This is because the effects of the matrix between the habitat patches on an organism's dispersal speed and survival can be explicitly incorporated into the calculation of connectedness (Ricketts 2001, Verbeylen et al. 2003). Least-cost modeling requires only two raster-based data inputs: (1) a 
resistance/friction layer indicating the travel cost through each cell, and (2) a source patch layer to which the cumulative travel cost for each cell is measured. The output is a raster cost surface indicating the effective distance from every cell in the landscape to a source patch. This analysis may be repeatedly run to virtually explore the connectedness of alternative design scenarios for nature reserves (Knaapen et al. 1992). The cost surface can be used to identify the least-cost path from any point on the landscape to a source. These least-cost paths may indicate the critical corridors that organisms use for transit or dispersal (Larkin et al. 2004) even if they are not necessarily the most likely routes organisms follow (Adriaensen et al. 2003). Least-cost path calculations have also been used to define functional distances between habitat patches in graph-based models of landscapes (Bunn et al. 2000). Most recent geographic information system (GIS) packages can perform least-cost calculations, a fact that has helped to promote the propagation of least-cost modeling as a conservation tool.

If connectivity analysis based on least-cost modeling is to be both reliable and credible, then the validity of input raster layers must be demonstrated. As is the case for any modeling exercise, the maxim "garbage in equals garbage out" holds true. For example, if resistance values are assigned to raster cells based on land-cover maps (Bunn et al. 2000, Meegan and Maehr 2002), then the land cover maps must be accurate and include features of interest to the organisms in question (Adriaensen et al. 2003). Similarly, the soundness of the least-cost calculations may be questionable if those resistance values can not be substantiated.

Least-cost modelers must also exercise extreme caution when using any GIS-based analysis of this kind. Technical issues associated with the rasterbased representation of spatial data may introduce errors in otherwise correct data that nullify results. In this paper, I address the potential presence of "cracks" in the resistance input layer of least-cost modeling exercises. Cracks result when narrow land features that are costly, i.e., those that are risky or speed-inhibiting from the point of view of a moving organism, such as roads or train tracks, are represented in raster form. These cracks lead to the erroneous identification of nonexistent "shortcuts" across truly expensive barriers. Several authors have correctly anticipated this problem and have recommended that linear features be buffered before tessellation (Adriaensen et al. 2003, Verbeylen et al. 2003). For example, Larkin et al. (2004) buffered major roads by $60 \mathrm{~m}$ before converting their maps to a raster format. There are four problems with this solution. First, when adding a buffer there is an implicit assumption that the area of influence of the linear feature is broader than its footprint. This assumption needs to be justified. Second, the empirical data or the expert opinion used to estimate the size of the zone of influence for linear features other than roads may be unavailable. Third, even nonlinear features may have narrow sections that develop cracks upon tessellation. Fourth, the buffers could potentially cover other important landscape elements (Adriaensen et al. 2003). For parallel, adjacent linear features, the less costly feature may vanish beneath the buffer of the more costly feature. Adriaensen et al. (2003) recommended that the grid cell size of the resistance layer be smaller than the narrowest element in the landscape. But this does not guarantee the absence of cracks, and even one crack could completely ruin the outcome of the analysis.

In this paper, I use a hypothetical example to explain resistance-layer cracks, how they occur, and the errors they generate with respect to least-cost modeling. I then present a simple algorithm to reveal the location of cracks and one approach to filling the cracks. Finally, the methods are demonstrated on a real data set. The negative repercussions of faulty resistance layers when conducting least-cost modeling can not be overstated. On the one hand, unimportant or nonexistent least-cost paths on a landscape may be mislabeled as important. As a result, conservation resources committed to protecting these paths will be wasted. Conversely, truly critical corridors may be overlooked, jeopardizing the organisms that rely on them.

\section{METHODS AND RESULTS}

\section{An example of cracks in a resistance layer for least-cost modeling}

Cracks in a resistance layer used as input for leastcost modeling are the effective gaps in narrow, costly landscape features that exist when these features are represented in a raster format (Fig. 1). In the absence of cracks, an organism crossing one of these features must "pay" the true cost associated with crossing. When cracks are present, the leastcost algorithm incorrectly finds inexpensive 
shortcuts across these features by permitting organisms to diagonally sidestep them. Unfortunately, the occurrence of cracks can not be reliably predicted. Linear features, such as roads or streams, are more likely to have cracks than nonlinear features. However, even nonlinear features may have narrow sections that exhibit cracks. Additionally, even extremely narrow costly features may not have gaps on a resistance surface if their orientation happens to be parallel to the $x$-axis or $y$ axis of the raster grid. As an example, consider a kidney-shaped wetland that is costly for organisms to cross relative to the surrounding landscape (Fig. 2 ). When the narrow west end of this wetland is parallel to the $x$-axis, the tessellation generates no cracks, and the correctly calculated least-cost path for an organism moving from south to north is around the west end of the wetland. If the wetland is rotated by $30^{\circ}$, the raster version of the wetland contains a crack. Now the least-cost path algorithm incorrectly calculates the least-cost path as passing through a crack across the narrow end of the wetland. A $60^{\circ}$ rotation produces even more cracks, and these also result in an error in the least-cost path calculation. After a $90^{\circ}$ rotation, on the other hand, the narrow section is parallel to the $y$-axis, and the cracks disappear. In this case, the least-cost path is correctly calculated.

\section{Finding the cracks}

The algorithm to identify the cracks in a resistance surface is essentially a neighborhood operation (Burrough and McDonnell 1998) in which the value of each cell, treated as the "central" cell, in the cost surface is compared with the values of bordering cells. The logic of the algorithm is as follows (see Figs. 3, 4, and 5):

If the north cell is equal to the east cell, and If the central cell is less than the north cell, and If the northeast cell is less than the north cell, Then the central cell is a type 1 crack.

Else, if the north cell equals the west cell, and If the central cell is less than the north cell, and If the northwest cell is less than the north cell. Then the central cell is a type 2 crack.

The values for the north, east, northeast, west, and northwest neighbors of the central cell

can be obtained by creating a series of shifted grids (Theobald 2000) using the "MakeIrregular" neighborhood function in the ArcView (Environmental Systems Research Institute 2000) spatial analyst extension. For example, the following Avenue script defines a three-by-three neighborhood and then yields the values of the northeast neighbors of the central cells:

firstLine $=\{1,0,0\}$

secondLine $=\{0,0,0\}$

thirdLine $=\{0,0,0\}$

theKernel $=\{$ firstLine, secondLine, thirdLine $\}$

theNbrHood $=$ NbrHood. MakeIrregular(theKernel $)$ theResult $=$ theGrid.FocalStats(\#GRID_STATYPE_MAX, theNbrHood,FALSE)

The map calculator and map query functions can then be used to execute the crack identification logic. For example, the following map query expression will highlight the type 1 cracks:

$([$ north $]=[$ east $])$ and $([$ central $]<[$ north $])$ and ([northeast] $<$ [north])

The crack type designation (type 1 or type 2 ) is used when the cracks are filled (see below).

\section{Filling the cracks}

A simple solution to eliminate the cracks in the resistance surface is to replace them with the value of a neighboring cell. The logic is as follows:

If the central cell is a type 1 crack,

Then replace its value with that of the east cell.

Else, if the central cell is a type 2 crack,

Then replace its value with that of the west cell.

The following map calculator expression will fill the type 1 and type 2 cracks (cell values for the type 1 or type 2 cracks are assumed to be set to a value of 1$)$ :

( [type 1]=1.AsGrid).Con([east], $(([$ type 2] $=1$. AsGrid).Con([west],[central] $))$ )

The crack-identification and crack-filling algorithms were successfully applied to the resistance surface from the kidney-shaped wetland example (see above) for the $30^{\circ}$ and $60^{\circ}$ rotations (Fig. 2). To demonstrate its effectiveness on real data, I also applied the crack identification algorithm to a resistance surface created from an 8000 ha section of an ecosystem map for the Resort Municipality of 
Fig. 1. A linear landscape feature represented in a raster format. The red line shows the feature. The black pixels show the raster representation of the feature. The dashed arrows indicated the location of cracks in the feature where organisms can diagonally sidestep across the feature (adapted from Fig. 4 in Adriaensen et al. 2003).

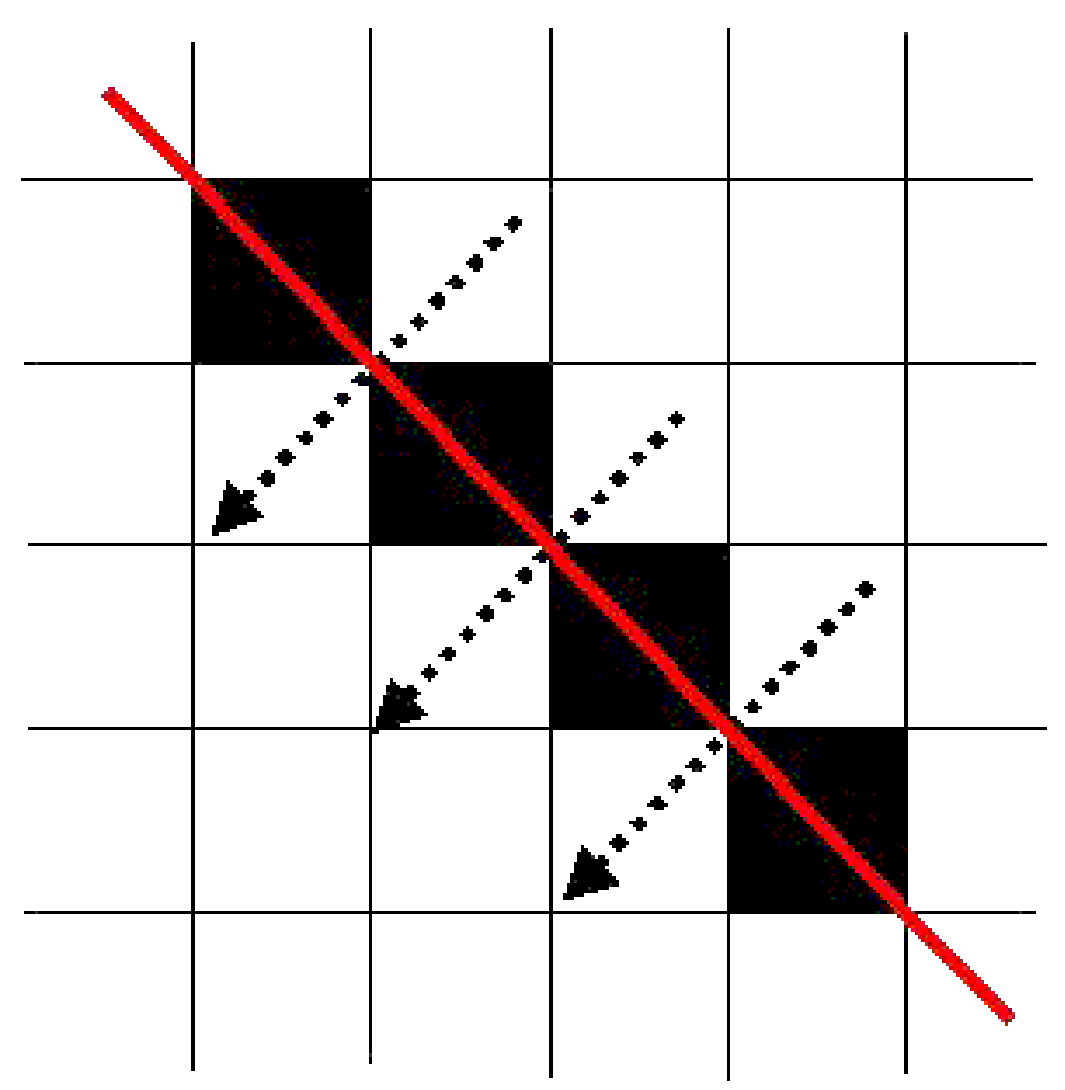

Whistler, British Columbia, Canada (Fig. 6; B. A. Blackwell and Associates 2004). Each polygon in the original map was given a resistance score based on the travel costs of a "large mammal" (Knaapen et al. 1992). The vector map was then converted to a raster resistance surface with $50 \mathrm{~m}$ pixels (163 rows and 195 columns $=31,785$ cells $)$. The crackidentification algorithm reveals that there are 423 cracks (250 type 1 and 173 type 2 ) scattered across the resistance surface section (Fig. 7). This represents $1.33 \%$ of the land surface.

\section{DISCUSSION}

Although only 423 of the 31,785 cells in the largemammal resistance layer were found to be cracks (Fig. 7), it is important to realize that just one crack can invalidate least-cost modeling calculations. The crack-identification algorithm presented here provides a simple way to scan resistance surfaces for these anomalies. There are cases in which cells could be incorrectly labeled as cracks.

For example, there may actually be a gap through a fence that is too small to register as an "open" cell. One solution is to run the crack-identification algorithm and then manually check the validity of each crack. The time necessary to perform this check 
Fig. 2. The green zones represent costly kidney-shaped wetlands surrounded by an easily traversable landscape. The red points represent the beginning and desired end points for a travelling organism. The first column shows the rotation of the wetlands relative to the original orientation $\left(0^{\circ}\right)$ of the wetland. The second column represents the same wetlands in a raster format where the blue lines show the least-cost paths between the red points. In the third column, red cells indicate the cracks (which would subsequently be filled). The dashed black lines indicate the original wetland outlines.

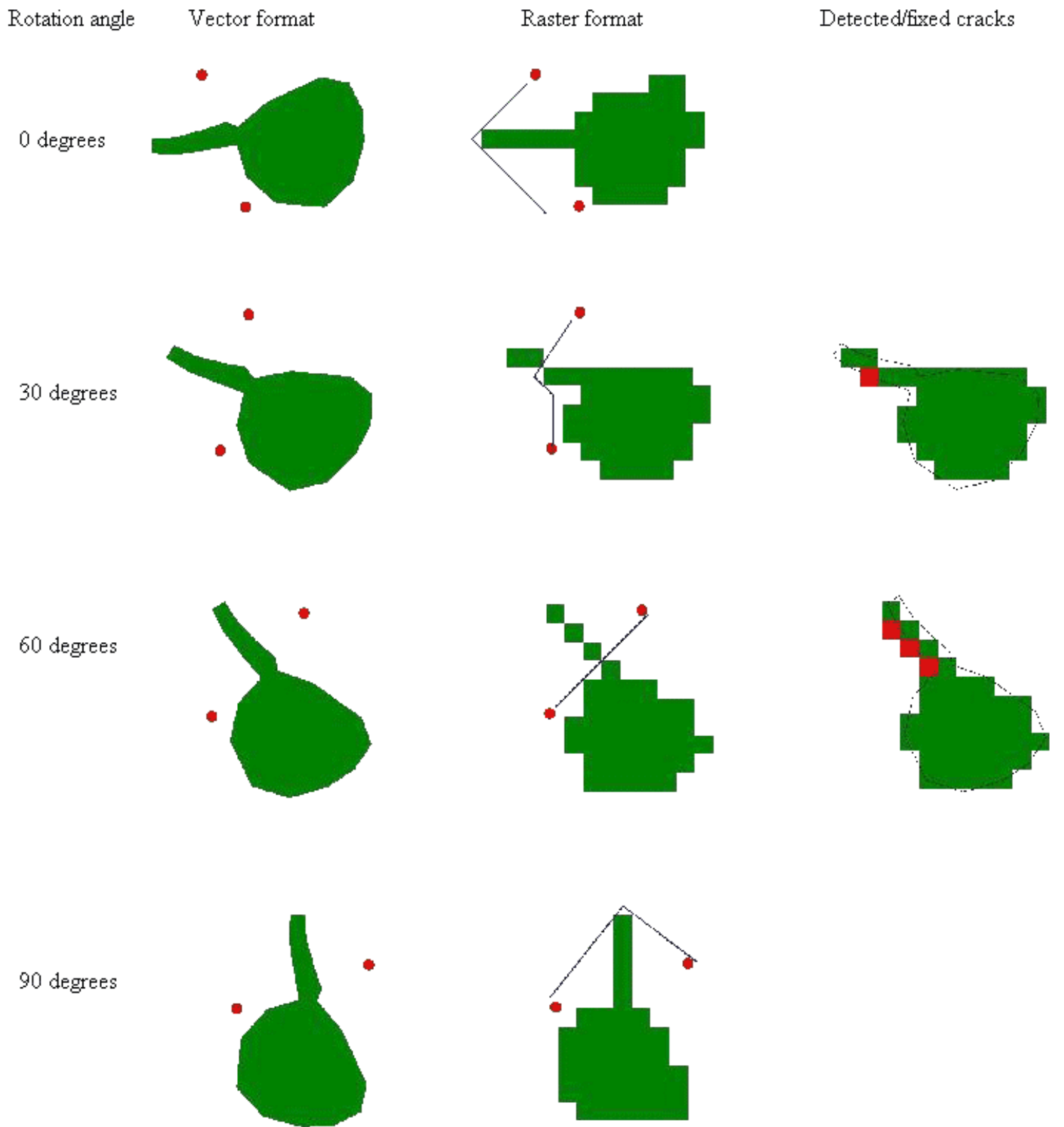


Fig. 3. Cell reference names for the crack identification and crack removal algorithms.

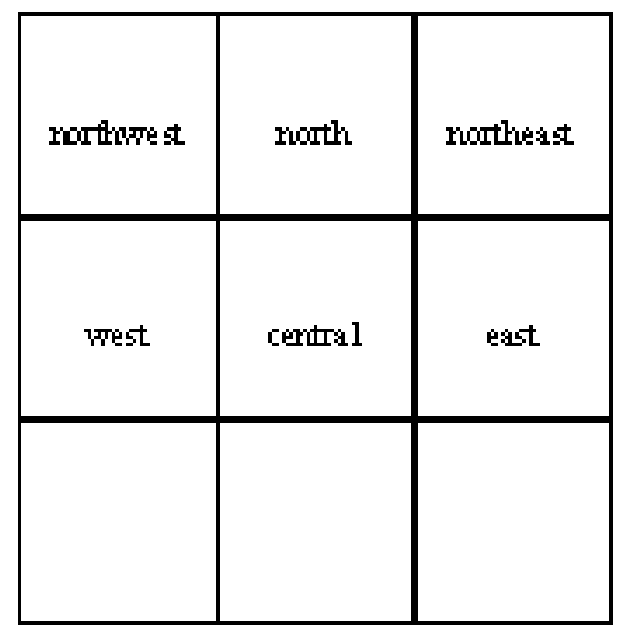

Fig. 4. An example of a type 1 crack. Shaded cells indicate the linear landscape feature. The arrow shows the crack though which organisms could diagonally sidestep across the feature.

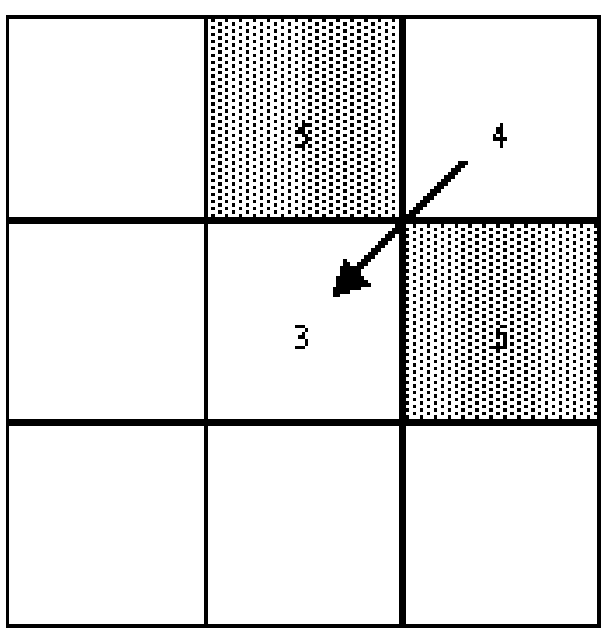

is small relative to the time necessary to undo errors that go unnoticed until later in the analysis. The crises facing many real-world conservation scenarios combined with the severe limits on time and monetary resources mean that conservationists must make quick decisions, often based on incomplete data. Nevertheless, the need for speed should be balanced against the time required to avoid needless mistakes.

Unlike the buffering method for dealing with cracks, in which narrow features are widened indiscriminately 
Fig. 5. An example of a type 2 crack. Shaded cells indicate the linear landscape feature. The arrow shows the crack though which organisms could diagonally sidestep across the feature.

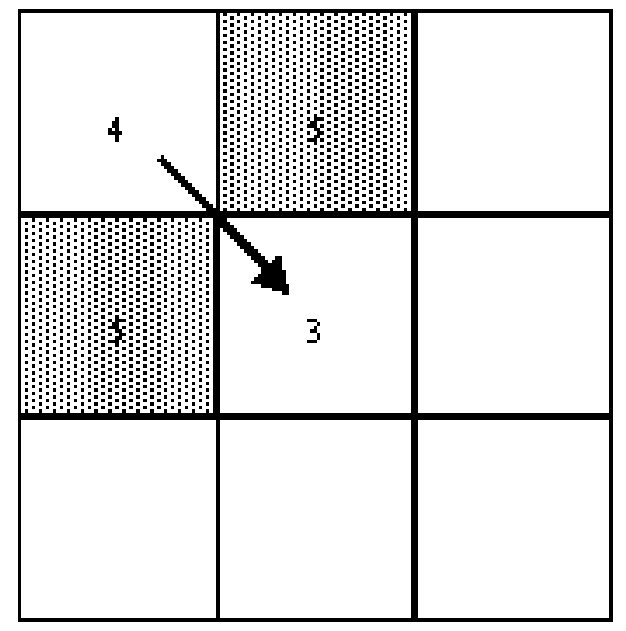

even when cracks are absent, the crack-filling algorithm results in minimal changes to the resistance surface. However, just as with buffering, the crack-filling algorithm could overwrite some important landscape features. One way to address this is to slowly rotate the resistance surface until its orientation shows the minimal number of cracks before running the filling routine. Another strategy for repairing the resistance surface that does not involve overwriting cell values is to repeatedly reduce the cell size of the resistance layers and rerun the crack-identification algorithm until there are no cracks. A third, more complicated approach is to switch all raster inputs to hexagonal rather than square pixels that eliminate the cracking problem but for which least-cost algorithms are less readily available.

The importance of the resistance layers to the outcome of the modeling requires that, as well as checking for cracks, least-cost modelers need to verify as much as possible the validity of the layers. Some modelers have calculated or had access to resistance values that were based on real habitat use (Ricketts 2001, Meegan and Maehr 2002). Regardless of the data source, sensitivity analysis is worthwhile (Adriaensen et al. 2003). For example, Larkin et al. (2004) and Knaapen et al. (1992) tested the robustness of their results to a rescaling of the resistance values. Verbeylen et al.
(2003) generated and tested 36 different resistance layers by varying the resistance values and the number of land-cover classes.

Although least-cost modeling has been empirically confirmed as an effective method for measuring isolation (Verbeylen et al. 2003), its use in the applied conservation field is relatively new. The advice contained in this paper is intended to support the continuing development of this highly valuable conservation tool. Along with refinements in the analysis methods, further empirical research is needed to accurately depict the effect of potential barriers on animal movement patterns (Orlando 2003).

Responses to this article can be read online at: http://www.ecologyandsociety.org/vollo/iss 1/art4/responses/

\section{Acknowledgments:}

I would like to thank the Resort Municipality of Whistler, British Columbia, Canada, and William de la Mare. 
Fig. 6. A resistance layer representation of an 8000 ha area in the Resort Municipality of Whistler, British Columbia, Canada. Darker colors indicate higher resistance values.

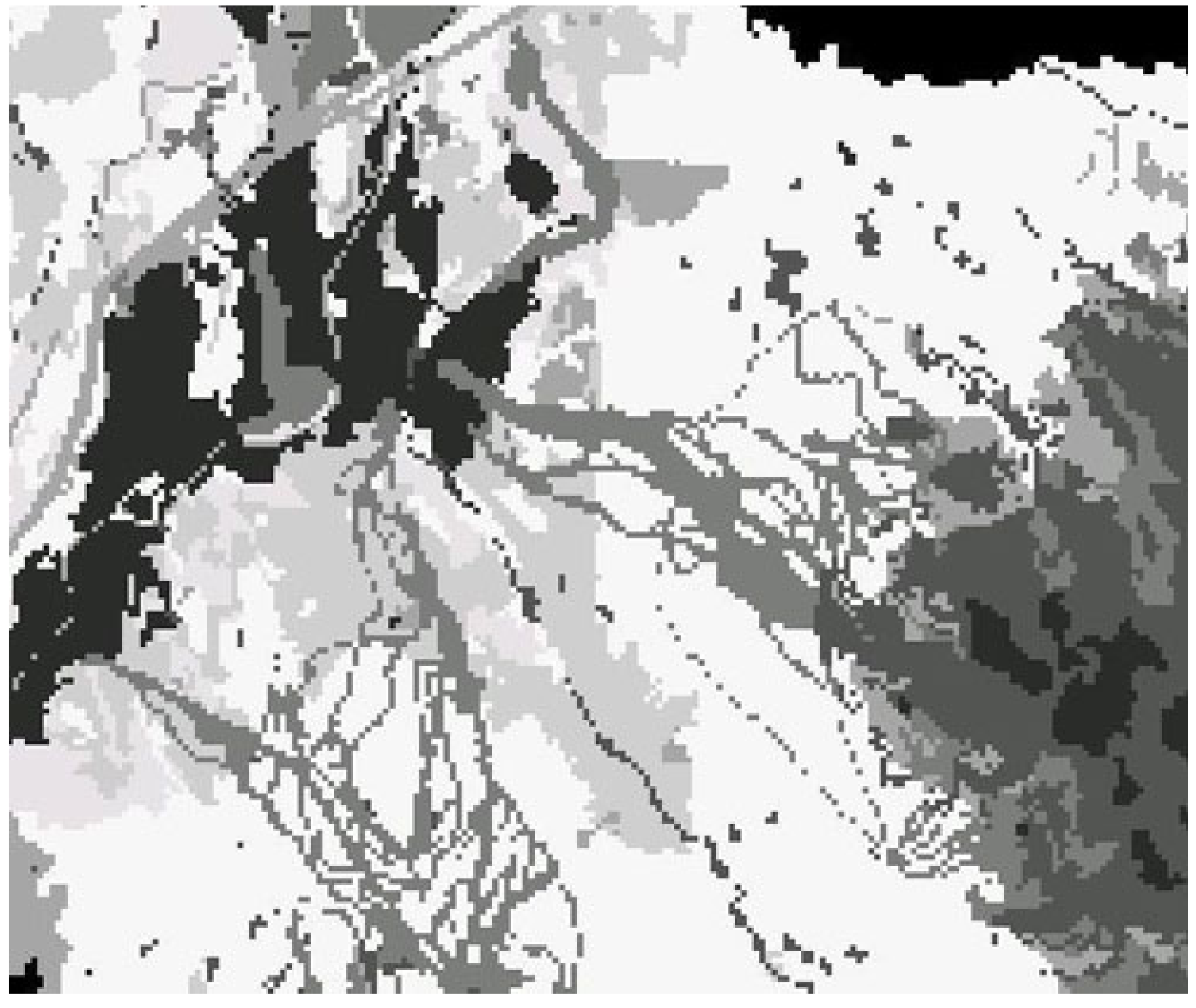

\section{LITERATURE CITED}

Adriaensen, F., J. P. Chardon, G. DeBlust, E. Swinnen, S. Villalba, H. Gulinck, and E. Matthysen. 2003. The application of 'least-cost' modelling as a functional landscape model. Landscape and Urban Planning 64:233-247.

Allendorf, F. W., and R. F. Leary. 1986. Heterozygosity and fitness in natural populations of animals. Pages 57-76 in M. E. Soulé, editor.

Conservation biology: the science of scarcity and diversity. Sinauer, Sunderland, Massachusetts, USA.

B. A. Blackwell and Associates. 2004. Terrestrial ecosystem mapping of the Resort Municipality of Whistler, North Vancouver, British Columbia, Canada. Resort Municipality of Whistler Municipal Council, British Columbia, Canada. 
Fig. 7. Cracks in the resistance layer representation of an 8000 ha area in the Resort Municipality of Whistler. Type 1 cracks are shown in red, and type 2 cracks are shown in blue.

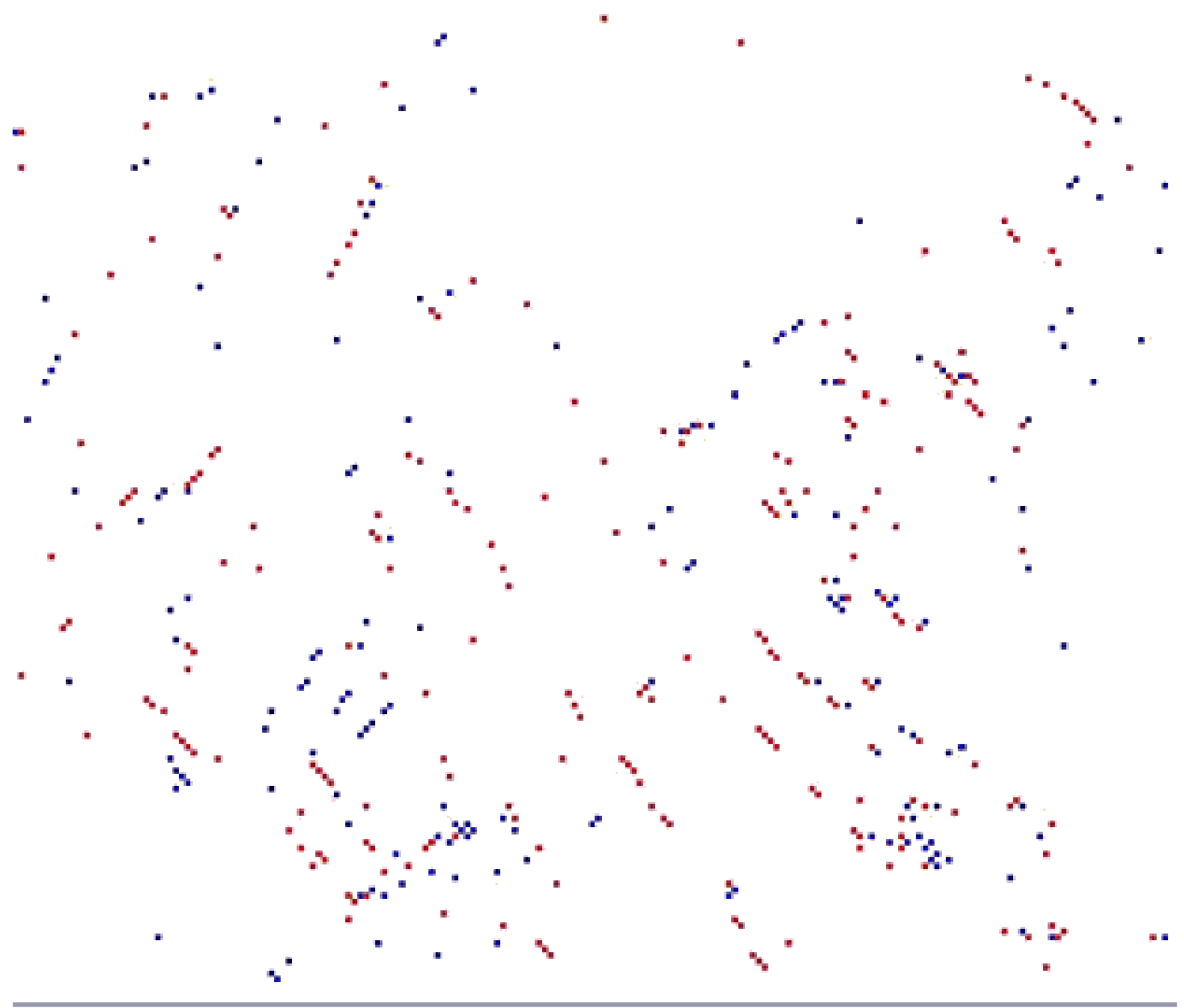

Briers, R. A. 2002. Incorporating connectivity into reserve selection procedures. Biological Conservation 103:77-83.

Bunn, A. G., D. L. Urban, and T. H. Keitt. 2000. Landscape connectivity: a conservation application of graph theory. Journal of Environmental Management 59:265-278.
Burrough, P. A., and R. A. McDonnell. 1998. Principles of geographical information systems. Oxford University Press, Oxford, UK.

Environmental Systems Research Institute. 2000. ArcView GIS 3.2a. ESRI, Redlands, California, USA.

Knaapen, J. P., M. Scheffer, and B. Harms. 1992. 
Estimating habitat isolation in landscape planning. Landscape and Urban Planning 23:1-16.

Larkin, J. L., D. S. Maehr, T. S. Hoctor, M. A. Orlando, and K. Whitney. 2004. Landscape linkages and conservation planning for the black bear in west-central Florida. Animal Conservation 7:23-34.

Meegan, R. P., and D. S. Maehr. 2002. Landscape conservation and regional planning for the Florida panther. Southeastern Naturalist 1:217-232.

Orlando, M. A. 2003. The ecology and behavior of an isolated black bear population in west central Florida. Thesis, University of Kentucky, Lexington, Kentucky, USA.

Ricketts, T. H. 2001. The matrix matters: effective isolation in fragmented landscapes. American Naturalist 158:87-99.

Soulé, M. E., and D. Simberloff. 1986. What do genetics and ecology tell us about the design of nature reserves? Biological Conservation 35:19-40.

Taylor, P. D., L. Fahrig, K. Henein, and G. Merriam. 1993. Connectivity is a vital element of landscape structure. Oikos 68:571-572.

Theobald, D. M. 2000. GIS concepts and ArcView methods. Third Edition. Conservation Planning Technologies, Fort Collins, Colorado, USA.

Tischendorf, L., and L. Fahrig. 2000a. How should we measure landscape connectivity? Landscape Ecology 15:633-641.

Tischendorf, L., and L. Fahrig. 2000 $b$. On the usage and measurement of landscape connectivity. Oikos 90:7-19.

Verbeylen, G., L. De Bruyn, F. Adriaensen, and E. Matthysen. 2003. Does matrix resistance influence red squirrel (Sciurus vulgaris L. 1758) distribution in an urban landscape? Landscape Ecology 18:791-805. 